\title{
Improving light microscopic detection of collagen by trichrome stain modification
}

\author{
S.S. Al-Mahmood \\ Department of Pathology and Poultry diseases, College of Veterinary Medicine, University of Mosul, Mosul, Iraq \\ email: saevan981@yhaoo.com
}

(Received October 13, 2019; Accepted December 12, 2019; Available online August 15, 2020)

\begin{abstract}
In present study we aimed to introduce modifications in classical protocol applied to stain collagen fibers by Masson's trichrome stain, in order to decrease time of different steps and concentration of chemicals in this traditional protocol. The experiment design contains a series of successive amendment steps based on previously successful steps, in which every step where be modified to get the best result, then the next step of staining protocol will be modified in the classical Masson's trichrome staining protocol. A tissue sections from liver, heart and intestines of sheep diagnosed with chronic fasciolosis were selected to be used in current study. The result showed that the new modified protocol gives the same staining efficiency to collagen fiber when Harris hematoxylin used as a nuclear strainer or even excluded this stain from staining protocol. In conclusion this new modified staining protocol give a perfect staining reaction to collagen fibers in pathological samples which is similar to that obtained by traditional Masson's trichrome stain, also this new modified procedure is less time consuming and less toxic effect on human and environment than other trichrome stains, and can be easily conducted either by the technician or the pathologists.
\end{abstract}

Keywords: Collagen, Modified, Trichrome, Improving, Histopathology DI: 10.33899/ijvs.2020.126176.1256, @2020, College of Veterinary Medicine, University of Mosul.

This is an open access article under the CC BY 4.0 license (http://creativecommons.org/licenses/by/4.0/).

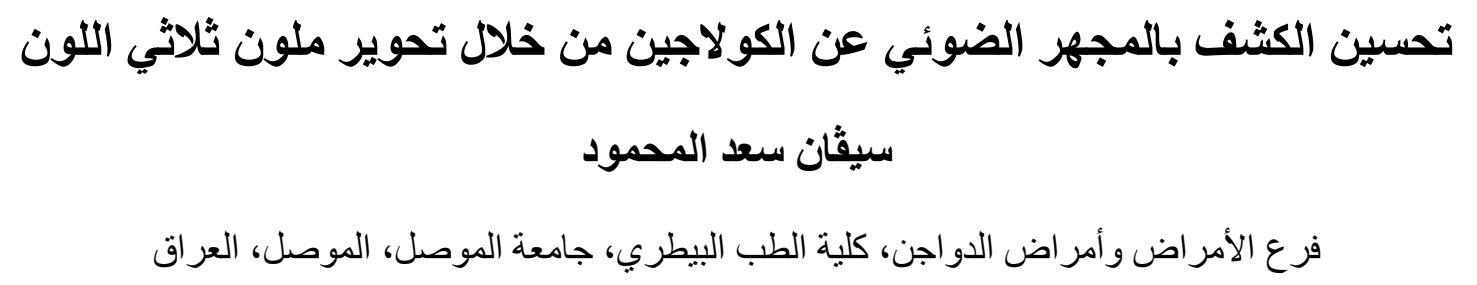

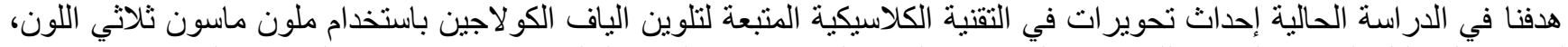

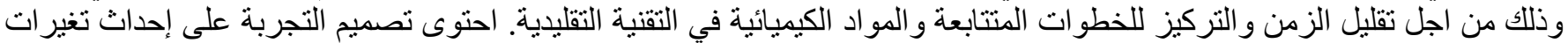

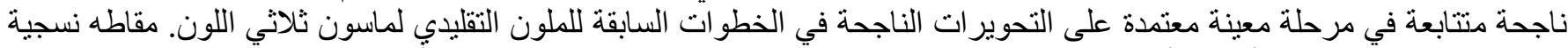

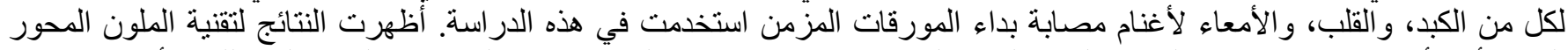

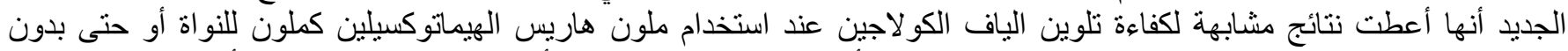

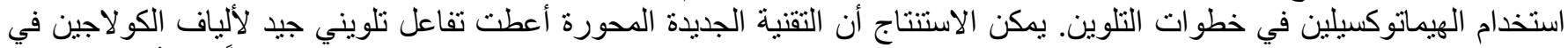

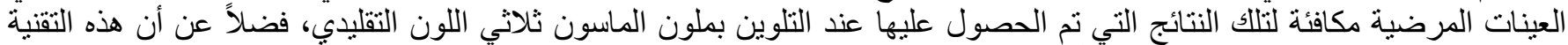

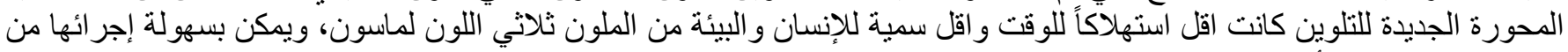




\section{Introduction}

Histology can be defined as the science that study the normal composition of tissues, while histological stains consider the key stone that used till now to explain different component of tissues (1). Histology and stains were invented and developed after a huge leap done by Antonie van Leeuwenhoek by invention the simple microscopy (2). Hematoxylin and Eosin stain (HE) and its modified protocols considered the main stain that applied routinely to visualize the components of tissues, in contrast, a special stain lead to expression a specific tissue component either in intracellular or extracellular location that could not clearly distinguished during HE routine staining (3). One of these components that need distinctive differentiation from surrounding cellular element is collagen fibers, these fibers are produced by mature fibrocytes and fibroblast in response to wide range of inflammatory cytokines produced by normal physiological and pathological process (4). The most common special stain used to facilitate the observation and identification of collagen fibers is trichrome stain, specifically Masson's trichrome stain, this stain and its modified protocols are widely used all over the global laboratories (4). Trichrome stain is invented by French scientist Claude L. Masson at 1910s, who used this stain to differentiate between collagen, muscle, and other fibers (3). Briefly, the original Masson's trichrome stain composed from four main stains, (i) Wiegert hematoxylin used to stain nucleus, (ii) Acid stain which target to stain muscles and red blood cells, (iii) Phosphatic acids that used to blech previous stains from collagen fibers and work also as mordent to anionic dyes that stain collagen in next step, and (iv) Collagen fiber stain (either Aniline Blue or Light Green SPF) that can be used to accomplish the goals of Masson's trichrome technique (3). Like all other special stains, this technique has undergone many modifications which aim to improving the accuracy and efficiency of collagen fibers staining (5). Since this special stain contain many toxic even carcinogenic chemical materials in addition time consuming protocol, this study designed to eliminate these hazardous chemicals and decrease their concentrations, in addition, to improve the detection of collagen fibers using a new modified technique of Masson's trichrome stain.

\section{Materials and methods}

\section{Tissue sampling and processing}

A tissue samples were collected from liver, heart and intestines of sheep diagnosed with chronic fasciolosis. The tissue samples fixed in 10\% neutral buffered formalin for at least 72 hours, then a represented sample were collected from area showed gross appearance of fibrosis, sliced at thickness less than $7 \mathrm{~mm}$, later these samples labeled with specific code, dehydrated with ascending concentration of ethyl alcohol, cleared in xylene, infiltrated and embedded with hot paraffine wax at $56^{\circ} \mathrm{C}$, later tissue blocks were sectioned at $6 \mu \mathrm{m}$ thickness, lifted at glass slides, attached to slide at $56 \pm 2^{\circ} \mathrm{C}$ for one hour, left to cool for another one hour, then cleared with xylene, rehydrated with descending ethyl alcohol to distilled water (6).

\section{Routine and special stain}

The histopathological glass slides from previous steps were stain by routine Harris hematoxylin and alcoholic red eosin (3) in order to confirm classical lesions of chronic fasciolosis and fibrosis, also the classical Masson's trichrome stain was used to stain slide which described by Layton and Bancroft (4) with Aniline Blue, slides with these stains considered as a control slides.

\section{Experiment designs}

The experiment design contains a series of successive amendment steps based on previously successful steps, in which every step where be modified to get the best result, then the next step of staining protocol will be modified in compare with the classical Masson's trichrome staining protocol.

Step one (mordent with Bouin's Solution) Bouin's solution used as a mordent for tissue samples fixed in formalin fixative (4), in this step the length of fixation with Bouin's solution will be decreased at a regular interval of 5 minutes in order to reach the least time for mordent which gives similar results to the control group.

Step two (staining the nucleus) Wiegert's hematoxylin used in Masson's trichrome stain in order to stain the nucleus (4), this stain will be modified in two manner (i) slides stained with Harris' hematoxylin instead of Wiegert's hematoxylin, and (ii) slides where not stained with hematoxylin stain.

Step three (staining with acidic stain) an equal mixed solution of $1 \%$ acid fuchsine and $1 \%$ biebrich scarlet where used as acidic dye in Masson's trichrome stain, in which the biebrich scarlet will stain the red blood cells, while acid fuchsine will stain muscle fibers (4), in this step the biebrich scarlet will be excluded from the staining steps, and the acid fuchsine will be decreased in concentration and staining time.

Step four (phosphate acids) both phosphomolybdic and phosphotungastic acid are the most commonly used in Masson's trichrome stain, they bind to collagen fibers and act as a site of conjunction with collagen stain that applied later (4), this step will be modified by decreasing the concentration and exposure time to phosphatic acids.

Step five (collagen stain) aniline blue is used to stain collagen fibers that conjugated with phosphatic acids, in which the anionic stain will attach to phosphatic acids 
rapidly due to negative charge induced by phosphatic acids (4), this step will be modified by decreasing the concentration and staining time to aniline blue.

\section{Evaluation of collagen fibers staining efficiency}

Tissue slides that stained in current study were examined by light microscope at 40x, using ToupView software version 1.0 to measure the intensity of collagen fibers staining (blue color). In brief, five similar fields in all slides from the same organ were subject to image color evaluating process, in which bright blue color were considered the positive result, then the image process calculated the mean of bright blue color in each field (7), later, the mean were statistical analyzed with aid of SPSS software version 19.0 at $\mathrm{P}<0.05$ using one-way ANOVA of Duncan's test (8).

\section{Results}

\section{Modified steps of trichrome stain (plates 1, 2, 3, and 4) Mordent with Bouin's Solution}

The result of this step showed that, decreasing in the time of fixation in Bouin's solution have a similar result to control slides when Bouin's solution preheated to $55^{\circ} \mathrm{C}$ and slides fixed for 15 minutes at $60{ }^{\circ} \mathrm{C}$, else that slides where fixed in Bouin's solution less than 15 minutes showed losing of brightness of collagen.

\section{Staining the nucleus}

The result of this step showed that, staining of slide with Weigert's hematoxylin or Harris hematoxylin or even without staining by hematoxylin stains did not affect the staining properties of collagen stain.

Instead, slides that not stained with hematoxylin stain are better in illustration of collagen fibers stained with Aniline Blue.

\section{Staining with Acidic stain}

The result of this step showed that, excluding of Biebrich Scarlet stain did not affect the staining properties of collagen or general architecture of stain, and reduction of Acid Fuchsine concentration to $0.1 \%$ for one minute will give the same result of muscle fibers and red blood cells as same as in control group.

\section{Phosphatic Acids}

The result of this step showed that, decrease the concentration of aqueous solution of Phosphomolybdic and Phosphotungastic acid to $0.5 \%$ for 5 minutes will give the same result of staining properties to collagen fibers that have been recorded in slides of control group.

\section{Collagen Stain}

The result of this step showed that, the concentration of $0.2 \%$ of aqueous solution of Aniline Blue give perfect staining properties to collagen fibers when used for one minute.

\section{Evaluation of collagen fibers staining efficiency}

The result of current study related to efficiency of collagen fiber staining showed that there are no significant differences between the slides stained with Masson's trichrome stain (control group) and slides stained with modified Masson's trichrome stain (experimental groups), these result indicate that the new modified staining protocol were have the same staining efficiency that obtained by classical protocol for Masson's trichrome stain (Table 1).

\section{Saevan's modified - Masson's trichrome stain protocol Solutions}

Bouin's solution prepared by mixing $75 \mathrm{ml}$ of formaldehyde $37 \%, 25 \mathrm{ml}$ of saturated aqueous solution of Picric acid, and $1 \mathrm{ml}$ of acetic acid (Glacial).

Table 1: Evaluation of collagen staining efficiency in control and experiment protocol

\begin{tabular}{lccc}
\hline & \multicolumn{3}{c}{ Mean \pm Std. error } \\
\cline { 2 - 4 } & Liver & Heart & Intestines \\
\hline Control Masson's trichrome & $83.18 \pm 2.05 \mathrm{a}$ & $64.40 \pm 1.97 \mathrm{a}$ & $18.74 \pm 1.37 \mathrm{a}$ \\
Modified stain without Hematoxylin & $83.05 \pm 0.99 \mathrm{a}$ & $64.13 \pm 3.70 \mathrm{a}$ & $18.52 \pm 1.52 \mathrm{a}$ \\
Modified stain Harris' Hematoxylin & $83.47 \pm 2.21 \mathrm{a}$ & $64.72 \pm 2.09 \mathrm{a}$ & $18.46 \pm 1.21 \mathrm{a}$ \\
Modified stain Weigert's Hematoxylin & $83.39 \pm 1.57 \mathrm{a}$ & $64.40 \pm 2.59 \mathrm{a}$ & $18.90 \pm 2.05 \mathrm{a}$ \\
\hline
\end{tabular}

Letters different in column have a significant difference at $\mathrm{P}<0.05$.

Aniline Blue stain prepared by mixing aniline Blue, 0.2 gram in $100 \mathrm{ml}$ of distilled water, the added $1 \mathrm{ml}$ of acetic acid (Glacial).

Acid fuchsine satin prepared by mixing acid fuchsine, $0.1 \mathrm{gram}$, in $100 \mathrm{ml}$ of distilled water. Harris hematoxylin stain prepared as describe by (3).
Phosphomolybdic - Phosphotungastic acid solution prepared by mixing 0.5 grams of phosphomolybdic acid, with 0.5 grams of phosphotungastic acid, in $100 \mathrm{ml}$ of distilled water, the added a $1 \mathrm{ml}$ of acetic acid (Glacial).

Aqueous acetic acid solution prepared by adding $3 \mathrm{ml}$ of acetic acid (Glacial), into $97 \mathrm{ml}$ of distilled water. 

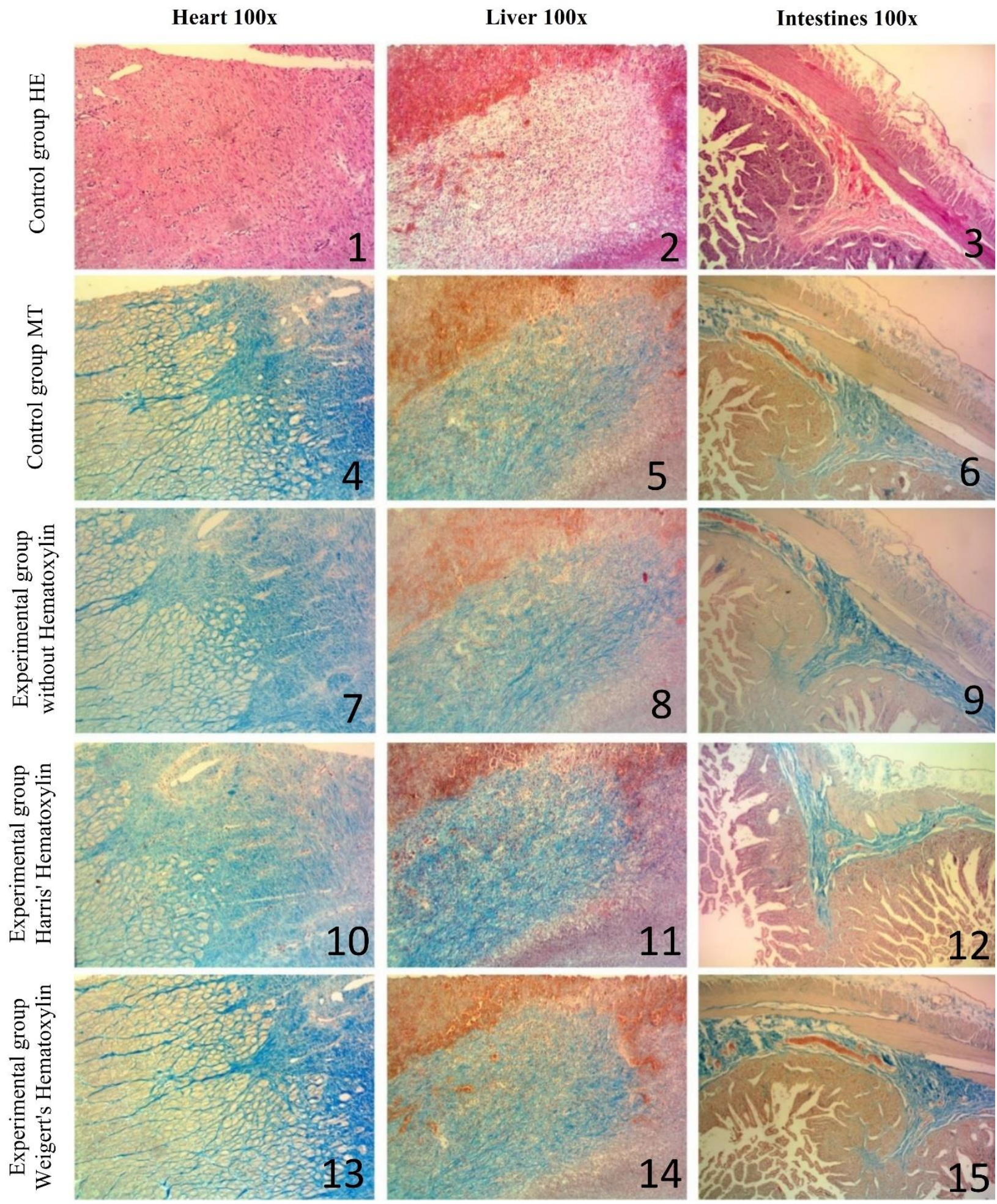

Plate 1: Showed histopathological comparison between the whole modified protocol of trichrome stain and traditional Masson's trichrome stain in liver, heart and intestines of sheep diagnosed with chronic fasciolosis. 

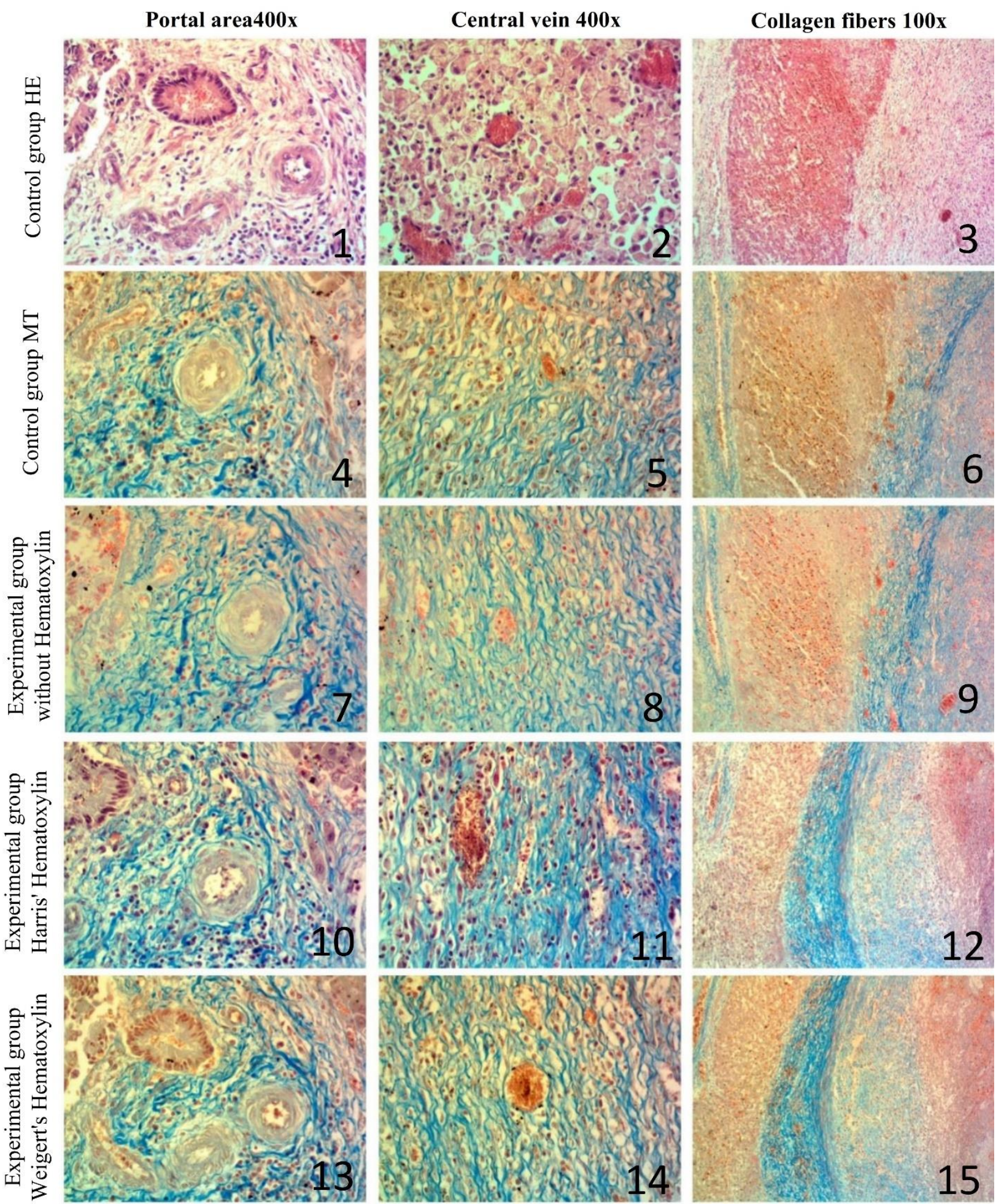

Plate 2: Showed histopathological comparison between the whole modified protocol of trichrome stain and traditional Masson's trichrome stain in liver of sheep diagnosed with chronic fasciolosis, in which the comparison conducted in (i) portal area, (ii) central vein, and (iii) collagen fibers. 

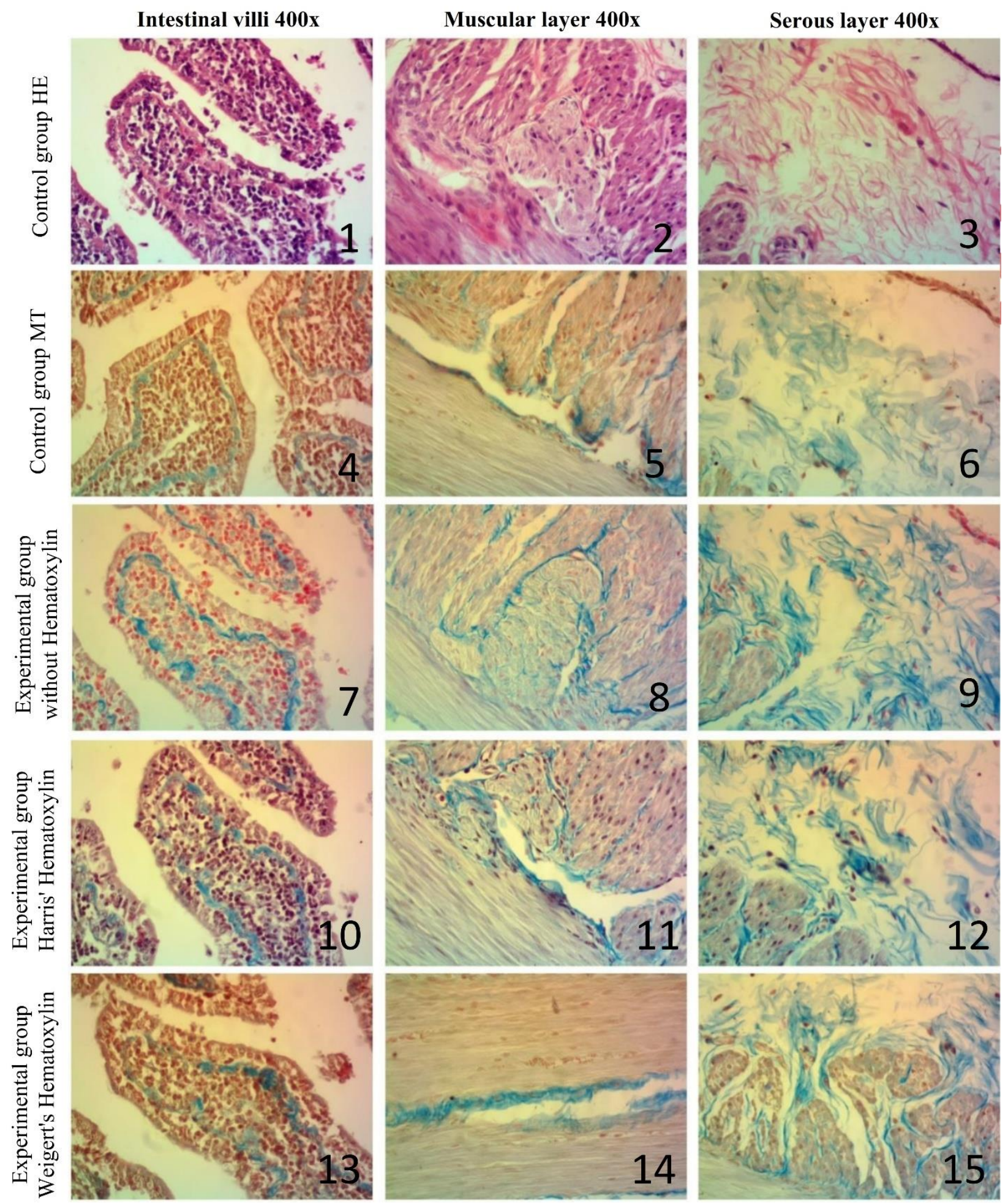

Plate 3: Showed histopathological comparison between the whole modified protocol of trichrome stain and traditional Masson's trichrome stain in intestine of sheep diagnosed with chronic fasciolosis, in which the comparison conducted in (i) intestinal villi, (ii) muscular layer, and (iii) serous layer. 

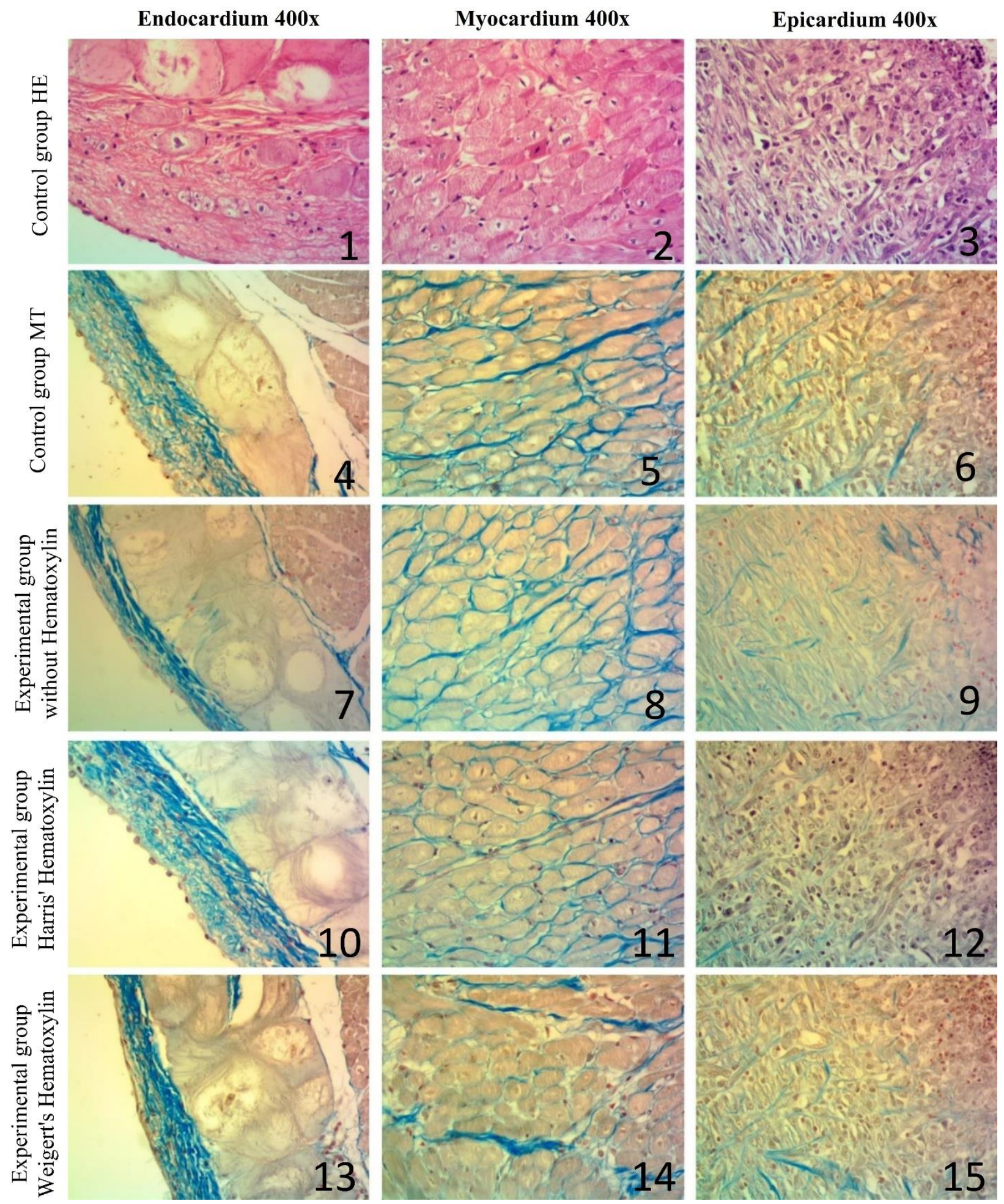

Plate 4: Showed histopathological comparison between the whole modified protocol of trichrome stain and traditional Masson's trichrome stain in heart of sheep diagnosed with chronic fasciolosis, in which the comparison conducted in (i) endocardium, (ii) myocardium, and (iii) epicardium. 


\section{Staining protocol}

Deparaffinized with xylol, three changes, 5 minutes each. Hydrate in ethyl alcohol start from 100, 90 and $80 \%$ for 5 minutes each. Complete hydration in tap water for 5 minutes. Preheat Bouin's solution at $55 \mathrm{C}^{\mathrm{o}}$, then mordent slides in heated Bouin's solution for 15 minutes at $60 \mathrm{C}^{\circ}$. Cool slides to room temperature then wash in running tap water till section loss the yellow coloration induced by Bouin's solution. Stain the nucleus using one step of these three options (a) Harris hematoxylin for 2 minutes, rinse in tap water, differentiate in acid alcohol for five quick dips, wash in tap water briefly, dip in ammonia water for few seconds, wash in running tap water for 10 minutes. (b) Weigert's hematoxylin for 5 minutes, then wash in running tap water for another five minutes. (c) If you don't use hematoxylin to stain nucleus omit this step. Rinse in distilled water. Stain in aqueous acid fuchsine solution for one minute, rinse quickly in distilled water. Phosphomolybdic / Phosphotungastic acid for 5 minutes, discharge the solution. Stain in Aniline Blue for one minute, then rinse quickly in distilled water. Aqueous acetic acid (glacial) for 5 minutes. Dehydrate slides in 80, 90 and $100 \%$ of ethyl alcohol. Clear in xylol three changes, 1 minute each. Cover slide with DPX.

\section{Color interpretation (plates 1, 2, 3, and 4)}

Collagen fibers, bright blue. Cell nucleus, sky blue (Harris' hematoxylin), or black (Weigert's hematoxylin), or colorless (without hematoxylin). Other tissue elements, red.

\section{Discussion}

Masson's trichrome stain consider the key role in differentiation of collagen fiber from other fibers including muscles (4). It is widely used to evaluated the presence and distribution of collagen fibers in diseases tissue as well as healing process, since routine hematoxylin and eosin stained all fibers (4). Many previous researches conducted to determine the amount of collagen deposition in tissues and their orientation in different stages of their production especially in wound healing and fibrosis process (9).

In current study we aimed to improve staining protocol of Masson's trichrome stain by exclude dangerous and carcinogenic chemicals, also decrease in concentration and staining time of these chemicals. The experiment conducted to decrease the mordant time showed that a fifteen minute in Bouin's solution at $60^{\circ} \mathrm{C}$ give a bright staining to collagen fibers in compare with slides of control group, treatment of tissue sectioned that fixed in formaldehyde solution with picric acid or mercuric chloride will increase the brightness and staining intensity of collagen fibers with aid of heat (10). Staining nucleus with counter stain using hematoxylin stain considered a key role in Masson's trichrome, since iron hematoxylin will affect the intensity of collagen staining but they improve general image of stained tissue, in addition iron hematoxylin will resists the bleaching induced by acidic solution that applied later (3), in contrast using of Harris hematoxylin will increase the intensity of collagen fibers appearance since it is stain nucleus with sky blue color, in addition exclude hematoxylin stain will not affect general staining image of trichrome stain (3), because the importance nuclear staining loss it effect when pathologist emphasizing on evaluation of collagen fibers deposition lesions more than importance of other tissue elements. Biebrich Scarlet considered a carcinogenic substance, and the importance of this chemical is to stain the RBC red (4). The result showed that reduction of Acid Fuchsine concretion will improve collagen staining conducted by current study experiments, since that the acid fuchsine will stain both collagen fibers and muscle fibers, so the reduction in staining time and concentration of Acid Fuchsin will decrease this side effect (11). Phosphomolybdic and tungstic acid considered as conjunction sit, in which these acids will bind with collagen fibers and Aniline Blue stain in next step (4). In current study the result showed that the least concentration of these acid was $1 \%$ for 5 minutes will give the same staining intensity in compared with control slides. Staining of collagen fiber by Aniline blue showed that the least concentration and staining were $0.2 \%$ for one minute will give a result similar to control slides.

Aniline Blue composed mainly from thiosulfate esrosaniline in di and tri form of phenyl, these di and tri phenyl have a great affinity to conjugate with phosphorus ions that binds to fibers especially in acid $\mathrm{pH}$, and this can be done by the aid of glacial acetic acid that added to aniline blue solution (12).

\section{Conclusion}

In conclusion this new modified staining protocol give a perfect staining reaction to collagen fibers in pathological samples which is similar to that obtained by Masson's trichrome stain, also this new modified procedure is less time consuming and less toxic effect on human and environment than other trichrome stains, which is easily conducted either by the technician or the pathologists.

\section{Acknowledgement}

This study was conducted in Veterinary Teach Hospital and College of Veterinary Medicine, University of Mosul, Mosul, Iraq.

\section{Conflict of interest}

The author declares that there are conflict of interests regarding publishing or funding this article. 


\section{References}

1. Brown PJ, Fews D, Bell NJ. Teaching veterinary histopathology: A comparison of microscopy and digital slides. J Vet Med Edu. 2016;43(1):13-20. doi:10.3138/jvme.0315-035r1

2. Larsson LI. Ctyology. In: Eurell JO, Frappier BL editors. Dellmann's textbook of veterinary histology. $6^{\text {th }}$ ed. Iowa: Blackwell Publishing; 2006. 1-16 p.

3. Layton C, Bancroft JD. The hematoxylin and eosin. In: Suvaran SK, Kayton C, Bancroft ID editors. Bancroft's theory and practice of histological techniques. $7^{\text {th }}$ ed. China: Churchill Livingstone; 2013. $174-185 \mathrm{p}$

4. Layton C, Bancroft JD. Connective and mesenchymal tissues with their stains. In: Suvaran SK, Kayton C, Bancroft ID editors. sBancroft's theory and practice of histological techniques. $7^{\text {th }}$ ed. China: Churchill Livingstone; 2013. 188- 189 p.

5. Iezzoni JC. Diagnostic histochemistry in hepatic pathology. In: Wikk MR editor. Seminars in diagnostic pathology. New York: Churchill Livingstone; 2018. 1-17p. doi: 10.1053/j.semdp.2018.10.003

6. Spencer LT, Bancroft JD. Microtomy: Paraffin and frozen. In: Suvaran SK, Kayton C, Bancroft ID editors. Bancroft's theory and practice of histological techniques. $7^{\text {th }}$ ed. China: Churchill Livingstone; 2013. 125- $127 \mathrm{p}$.

7. Gurcan MN, Boucheron LE, Can A, Madabhushi A, Rajpoot NM, Yener B. Histopathological image analysis: A review. IEEE Rev Biomed Eng. 2009;2:147-171. doi:10.1109/RBME.2009.2034865

8. Handel IG. Statistics for veterinary and animal science. $3^{\text {rd }}$ ed. New York: Wily Blackwell; 2013. 12-78 p. doi: 10.1136/vr.f7415

9. Noorlander ML, Melis P, Jonker A, Noorden CJ. A quantitative method to determine the orientation of collagen fibers in the dermis. $\mathrm{J}$ Histochem Cytochem. 2002;50(11):1469-1474. doi: $10.1177 / 002215540205001106$

10. Rhodes A. Fixation of tissue. In: Suvaran SK, Kayton C, Bancroft ID editors. Bancroft's theory and practice of histological techniques. $7^{\text {th }}$ ed. China: Churchill Livingstone; 2013. 87 p.

11. Lillie RD, Tracy RE, Pizzolato P, Donaldson PT, Reynolds C. Differential staining of collagen types in paraffin sections: A color change in degraded forms. Virchows Arch Pathol Anat Histol. 1980;386(2):153-159. doi: $10.1007 / \mathrm{bf00427227}$

12. Cima L, Riva G, D'Errico A, Casartelli M, Capelli P, Tomezzoli A, Eccher A. Fast Chromatrope Aniline blue special stain is a useful tool to assess fibrosis on liver biopsy during transplantation. Transplant Proc. 2017;49(4):667-670.doi: 10.1016/j.transproceed.2017.02.024 